\title{
Digital Images in United States and Canadian Dental Education: The Role of Delivery Medium and Metadata
}

\section{Stephen Paling}

Library and Information Studies, University at Buffalo, State University of New York, 534 Baldy Hall, Buffalo, NY 14260-1020 swpaling@buffalo.edu

\section{Melissa Miszkiewicz}

Academic Computing \& Technical Services, Buffalo State College, Buffalo, NY 14222 miszkimj@buffalostate.edu

\section{June Abbas}

Library and Information Studies, University at Buffalo, State University of New York, 534 Baldy Hall, Buffalo, NY 14260-1020 abbasjm@buffalo.edu

This is the first of two papers that will describe the outcome of a needs assessment that focused on the image-related needs of dental faculty members and clinicians. The needs assessment was conducted in 2005 at the University at Buffalo School of Dental Medicine. It addressed two specific goals: to begin examining whether online delivery of digitized dental images would match the needs of dental faculty members and clinicians, and to begin examining what type of image metadata would best match the needs of those faculty members and clinicians.

\section{INTRODUCTION}

In support of an electronic curriculum, health sciences educators and administrators are becoming aware of the need for digital repositories to catalog, store and provide access to digital images and other digital learning objects (Fleiszer, Posel, \& Steacy, 2004). Tsafrir and Ohry, among others, note that technology has continually changed the way in which images are captured and delivered and that the increasing "accumulation of pictorial material has posed considerable problems of storage, cataloging, retrieval, display and dissemination of the information"(Tsafrir \&Ohry, 2001, p. 99). Other researchers, such as Attig, Copeland, and Pelikan (2004), have examined the challenges of creating metadata 
for a library of digitized images.

Images are a primary tool used across the medical curriculum to communicate information and share content when teaching and practicing medicine (Fiuza, de Padua, $\&$ Lopes, 1997). Less is known, though, about the use of images in dental teaching. While a number of articles have addressed the information needs of medical professionals, relatively few articles have addressed the needs of dental professionals. Lawrence and Levy (2004), for example, conducted a study of the searching knowledge of medical and dental students, but their study did not directly address the description, organization, and use of digitized dental images. This leaves a gap in the research that this needs assessment sought to address.

Access to information technology across U.S. and Canadian dental schools is increasing. Results from the first phase of an ongoing study of Institutional Readiness for Electronic Curriculum (IREC), in June 2003, led by the University of Texas Health Science Center at San Antonio, Office of Educational Research and Development makes evident that “...the majority of dental schools have provided their faculty and students with numerous instructional technology (IT) applications and capabilities" (University of Texas Health Science Center at San Antonio, 2003, p. 4). Likewise, eighty-five percent (85\%) of all dentists are using computers in their practice (Schleyer, Spallek, Bartling, \& Corby, 2003, p. 4).

Like their colleagues throughout the country, faculty at the University at Buffalo School of Dental Medicine have amassed thousands of dental and medical images in the form of slides and videotape, and use them daily in lecture presentations, clinical and preclinical teaching. Anecdotally, it would seem that several developments both internal and external to the School of Dental Medicine have caused faculty members and clinicians to prefer digital images at an increased rate. From a general perspective one can consider these developments to include access to computers, scanners and projectors, presentation software, web editing/publishing software, image augmentation software, animation software and video production software along with staff to assist with their use in conversion and production. Additionally, the discontinued manufacture of traditional slide carousel projectors may have created both the desire and need for digital images. However, the focus in previous research on medical image use, rather than dental image use, leaves a gap in our knowledge of what remaining image-related needs dental faculty members and clinicians have.

To begin filling that gap, this paper reports part of the outcome of a needs assessment conducted as part of preparing for construction of a possible online repository of digitized dental images. The portions of the needs assessment reported here will focus on two 
goals: to begin examining whether online delivery of digitized dental images would match the needs of dental faculty members and clinicians, and to begin examining what type of image metadata would best match the needs of those faculty members and clinicians.

This paper is a follow-up to Paling and Miszkiewicz (2005), and a companion piece to Paling, Miszkiewicz, Abbas, and Zambon (in progress), which will cover the general image use needs of dental faculty members and clinicians separate from delivery medium or metadata.

\section{Background}

Access to digital image collections has traditionally been possible through two techniques: content-based indexing and concept-based indexing. Research in these two areas has dominated image retrieval research, with a discipline-specific division between the two techniques. Chu (2001), through a scientometric study of citations on image retrieval research, discovered that "people in the field of computer science focus on the content-based approach, while the information science community, including library science, concentrates on the description-based [concept-based] method" (p. 1011). One benefit of the results of the needs assessment discussed in this paper will be preliminary indications of which of these techniques will best match the needs of dental faculty members and clinicians.

\section{Content-Based Retrieval}

Content-based retrieval focuses on the automatic extraction and identification of features of the image such as color, shape, or texture. Content-based indexing was seen as a way to overcome the limitations of concept-based indexing by automatically identifying the indexable features of an image. Narasimhalu (1995) and Gupta and Jain (1997) defined the field with their research on computer-based interpretation systems, while Gudivada and Raghavan (1995) led the way with lists of query classes (or attributes) that can be used by those systems. Examples of these classes are: color, texture, sketch, shape, volume, spatial constraints, browsing, and objective attributes. Classes such as color and shape are suggestive of possibilities for dental images. For example, the color and shape of a lesion can constitute important diagnostic information.

The majority of image retrieval research currently is focused on developing more robust content-based systems of image retrieval employing advanced techniques such as querying by visual contents using spatial arrangement and chromatic attribute similarity 
(Corridoni, Bimbo \& Vicario, 1998), and shape feature extraction methods (Li, Lee \& Adjeroh, 2005) to name a select few. Comprehensive discussion of content-based retrieval is beyond the focus of this paper, and the research much too numerous to report in this space. The research covered in this section, though, is sufficient to establish the context of the current study. The reader is referred to Del Bimbo, 1999; Smeudlers, Worring, Santini, Gupta \& Jain, 2003; Smith, 2001; Zachary \& lyengar, 2001 and Li, Lee, \&Adjeroh, 2005 for more comprehensive coverage of the general problems and techniques of content-based retrieval systems.

\section{Concept-Based Retrieval}

Rasmussen (1997) defines concept-based indexing as a system which manually identifies and describes images and the objects therein, in terms of what they are and what they represent. Concept-based indexing uses traditional text indexing schemes such as controlled vocabularies or natural language processing. For example, concept-based image retrieval employs a specialized controlled vocabulary which emphasizes unique fields or concepts relevant to the user group. Early research into concept-based image retrieval was focused on art collections and the development of specialized controlled vocabularies to use for indexing the images. Examples of these indexing vocabularies are 1) ICONCLASS which was developed between 1973 and 1985; 2) Art and Architecture Thesaurus (AAT) which was created in 1980 and now is supported by the Getty Information Institute; and 3) the Library of Congress Thesaurus for Graphic Materials (TGM I) which was developed to catalogue the Library of Congress' Prints and Photographs Division (Rasmussen, 1997 pp. 179 - 180). A controlled vocabulary for dentistry (SNODENT) exists. It offers some possibilities for application for digitized dental images delivered online. However, it has not yet seen widespread use (Goldberg, Ceusters, Eisner, \& Smith, 2005).

A second type of concept-based retrieval uses the natural language or textual information (free text captions), which often accompany images, as access points. This indexing technique may also be used in conjunction with controlled vocabularies. In this area, Turner (1995) is known for his pioneer work in applying free-text descriptions to photographs. Rowe (1994) worked with the stored image captions of aircraft and weapons images, while Shihari and Rapaport (1990) used the linguistic information from captions with pictorial information to identify photographs. Ornager (1997) takes it a step further and incorporates semantic analysis into her study. She explored how newspaper journalists requested and searched for images by utilizing caption information. Her study is also significant in that it is one of the first user studies on image searching. 
A third but emerging area of concept-based indexing uses descriptors or terms provided by the users themselves. This sociocognitive-based method incorporates cognitive, social, and affective states or attributes into the descriptors or indexing of an image. This strategy is generally more user-centered and takes into account the communication, social, and individual constraints and contexts of both the image and the searcher. Hastings (1995; 1997) explored the use of user-supplied descriptors to digitized art images; O'Connor, 1996; O'Connor, O'Connor, \& Abbas, 1999; had users supply captions, subject headings, and annotations to a collection of digital images. Turner's (1997) research further supports the use of user-assigned terms for storage and retrieval of moving images. This possibly could include annotations or case studies from contributors of dental images.

\section{Image Searching}

A further area that must be mentioned is that of image searching studies. This line of research informs system design of both content-based and concept-based systems. While there are few image searching studies to date, and even fewer Web-oriented image searching studies, this research area is growing in popularity. Keister (1994) conducted one of the earlier studies of a medical image collection. She used the National Library of Medicine's Prints and Photographs Collection to examine queries of users. Rowe (1994) utilized syntactic and semantic linguistic clues in captions to search for images in a database of aircraft and weapons images. Hastings (1994) explored the retrieval issues encountered by art historians using art image databases. Hastings (1995) analyzed the queries art historians used to retrieve images from a select collection of Caribbean paintings which had been digitized. Hastings identified four levels of query complexity based upon the focus of the search terms. Ornager (1997) explored how newspaper journalists requested and searched for images by utilizing caption information. She utilized a word association methodology to determine common descriptors used by the journalists. She then developed a typology of the photos and the descriptions used to retrieve the photos. Fidel (1997) explored how the nature of the retrieval task or the intended use for the image can affect how searchers may proceed in the search task. She posits that there is a continuum on which images reside. Jorgensen (1996) explored the use of a template for describing images. She requested inexperienced searchers to fill in a predefined template when conducting a search session for images. Jorgensen later used the terms to assign descriptors to the images for use in future retrieval. Subsequent studies by Jorgensen (1998); Jorgensen, Jaimes, Benitez, \& Chang (2001); Jorgensen and Jorgensen (2003b); have identified common attributes used while users searched for images, and have tested these attributes in further research. Armitage and Enser (1997) 
in one of the largest image searching studies, collected user's queries and requests for images from seven picture libraries. The collections of the participating libraries represented both general and special collections which supported a wide spectrum of users. Enser and Armitage identified four major types of queries, 1) image content, 2) identification or reference checking, 3) accessibility of images, and 4) miscellaneous non-image related queries. Their research demonstrates the presence of similarities between image queries and query formulation across a wide spectrum of libraries and users. Several of the query types are suggestive for the current needs assessment. For example, image content and reference checking both could play a role for users of dental images who want images depicting particular areas of the maxillofacial region (image content), or who want to verify a diagnosis (identification). Choi and Rasmussen (2003) reviewed queries of faculty and students in American History using the American Memory Digital Library collection as a test bed. The study found that subject related terms, as well as format terms were used often, and author and title searches were rarely found.

\section{Image Searching on the Web}

Few studies to date focus on searching for images on the Web. Mandelbaum (1994) researched how users search for pictures in full-text on-line newspapers. Jasco (1998a) looked at how users search for video on the Web. Jasco (1998b) addressed how users search for Web images utilizing concept-based search engines. Goodrum and Spink (2001) analyzed image queries made to the EXCITE search engine. Pu (2003) compared queries of image and textual Web searches for feature length and frequency. He also classified image and textual queries into broad subject categories to compare popularity of terms.

\section{Metadata Scheme Development Based on User Studies}

A final area of research that is directly related to this paper, but also an understudied area is that of specialized metadata scheme development based on user studies. To date little extant research has been reported in this area. Jorgensen, 1996; 1998, as reported above, identified and tested common attributes used while users searched for images. These common attributes include: of object, person, social status, color, body part, location (specific), and activity. She later categorized them into three broad categories of perceptual, interpretive, and reactive. Her image template is useful for indexing and searching of images. This is another area that is suggestive for the current needs assessment. Attributes such as color (e.g., of a lesion) and body part have clear 
application to dental images.

\section{Methodology}

Data were gathered in two stages for the needs assessment. The first stage incorporated semi-structured interviews with dental faculty members and clinicians on the faculty of the University of Buffalo's School of Dental Medicine. The School provides a comprehensive dental curriculum that fairly represents the curriculum and methods in use at other American dental schools. The second stage incorporated a brief survey of academic deans at dental schools around the United States to provide national context.

The sample for the needs assessment was purposive, and was designed to cover a broad range of opinion. The sample included faculty members from a variety of dental disciplines, as well as faculty members who teach general medical courses such as physiology that are also conducted under the auspices of the School. The clinicians were also drawn from a variety of specialties.

The academic deans at American dental schools were invited to participate through the listserv of the American Dental Education Association (ADEA).

\section{Interviews with Faculty Members and Clinicians}

The interviews were intended to yield useful evidence for both of the goals discussed in this paper: to begin examining whether online delivery of digitized dental images would match the needs of dental faculty members and clinicians, and to begin examining what type of image metadata would best match the needs of those faculty members and clinicians. The first goal was served by discussing with dental faculty members and clinicians whether and how they use online resources generally, which provided useful context for the online delivery of dental images. The second goal was served by discussing more specifically with the dental faculty members and clinicians how they use images and what they use images for, which provided useful context in deciding whether and how a metadata scheme might aid their retrieval and use of online dental images.

The interviews included both brief objective questions to establish relevant context, as well as semistructured questions mean to elicit richer information about the respondents' use of online resources in general and digitized dental images in particular. The interviews were all in-person and conducted on the campus of the University at Buffalo.

The objective portions of the interviews included brief questions about which online 
resources each respondent used in his or her professional efforts, and whether each respondent made professional use of dental images. This directly addressed the first goal of determining whether online delivery of digitized dental images would match the needs of the respondents.

The data from the objective questions were used to derive descriptive statistics that will be discussed in the Results section. Respondents were asked whether they used each of the following resource types, which are listed along with bracketed abbreviations used here in charts and tables:

- [Engine] Internet search engines, e.g., Google.

- [UnivRes] Online dentistry resources maintained by the University, e.g., a dentistry-related bulletin board.

- [ UnivRes] Online dentistry resources not maintained by the University, e.g., DERWEB.

- [Dbase] Online databases, e.g., MedLine.

- [CD/DVD] Information on CD-ROM or DVD, e.g., the Stanford Tooth Atlas.

- [Other] Other information, e.g., information delivered through a PDA.

- Dental images similar to those in a set of sample images. Abbreviated as follows:

- [Image] Images used without specifying whether the images were digital or non-digital.

- [Diglmage] Use of images in digital form.

- [ Diglmage] Use of images in non-digital form.

The semistructured interview questions asked each respondent to indicate the circumstances under which the respondent used each resource that he or she used, and what hindrances, if any, the respondent had encountered in using each of those resources. Before the final set of semistructured questions each respondent was shown the same sample of dental images that were meant to represent the range of images that might be included in a digitized collection of dental images. That set of semistructured questions then asked each respondent to indicate whether, and under what circumstances, the respondent used dental images similar to the samples. The respondents were also asked what hindrances, if any, they had experienced in using dental images. This directly served the second goal by establishing the context and type of use that a potential metadata scheme could be designed to aid. The semistructured components of the interviews allowed for unanticipated, productive factors to emerge.

The semistructured components of the interviews were recorded and transcribed, and the research team performed a content analysis of the transcript data from the semistructured questions. The content analysis involved two stages. 
In the first stage, statements from the interviews were grouped by the resource from the list above to which each statement referred. In many cases this was very clear based on the question. However, the semistructured nature of the questions meant that the interviews occasionally moved between multiple subjects. That made it necessary to verify that the researchers concurred in coding the resource to which a respondent was referring. The first content analysis scheme was developed to allow this.

In the second stage, the statements grouped under each resource type were further grouped into the following categories:

- Prompt: the statement referred to the conditions or reasons that prompted (or hypothetically might prompt) a respondent to use that resource type.

- Hindrance: the statement referred to a condition that in some way hindered the use of a particular resource type, or prompted the respondent to not use that resource type.

- Description: The statement listed or referred to a resource type without elaborating about any prompt or hindrance.

Because of the relatively short list of categories in each scheme, chance agreement presented a potential problem with both. Cohen's Kappa was used as a tool to correct for that potential problem (Cohen, 1960, p. 41). Carletta (1996) provided rules of thumb for interpreting Kappa. A score of greater than .67 allows preliminary conclusions to be drawn, and a score of .80 or greater is considered an indicator of good reliability (p. 252). Research team members were able to reach a score of .80 with both content analysis schemes. The outcome of that content analysis is discussed in the Results section. Grouping the statements into two sets of categories also permitted the use of descriptive statistics, which were appropriate for a purposive sample. Those statistics are discussed as part of the results.

\section{Survey of Academic Deans}

The survey of academic deans provided important context and validation for the interviews with dental faculty members and clinicians. Academic deans are in a good position to know what resources their faculty members use, and the survey asked the deans to indicate whether their faculty members use dental images in general, and digitized dental images more specifically, in their work. That information provided a national context for the use of such images beyond the University at Buffalo's School of Dental Medicine. The academic deans were asked the following questions: 
1. Do your faculty use digital images to teach dentistry?

2. If so, what resources provide images for faculty use? Please check all that apply:

3. Free sources on the Internet.

4. Personal faculty collections.

5. Subscriptions such as DERWEB.

6. Digital textbooks.

7. Digital journal articles

8. Commercial DVDs or CD-ROMs.

9. Do you believe that your faculty would benefit from and utilize a copyright free source of digital images in oral pathology/oral medicine and histology available for desktop delivery via the Internet?

\section{Results}

Interviews with Faculty members and Clinicians

The research team conducted at total of 16 interviews with faculty members and clinicians at the University's dental school. The sample included respondents in a variety of dental disciplines, as well as respondents in general medical disciplines who work within the dental school. The sample skewed heavily male, with 11 male respondents and 5 female respondents.

In the objective section of the interview, each respondent was asked whether he or she used online resources in the categories listed in the last section. Table 1 shows the results by resource type, except for images, which will be discussed separately. All six of the resource types were used by multiple respondents, with 4 of the 6 resource types being used by at least 12 of the 16 respondents (75\%).

Table 1: Frequency of Resource Use by Type

\begin{tabular}{|l|c|c|c|c|c|c|}
\hline Frequency/Resource Type & Engine & UnivRes & UnivRes & Dbase & CD/DVD & Other \\
\hline Frequency $(\mathrm{n}=16)$ & 15 & 13 & 7 & 13 & 12 & 5 \\
\hline
\end{tabular}

Table 2 breaks down the results by respondent number (" $x$ " indicates use of a resource type, with images omitted). Of the six online resource types, each respondent used at least two, and 10 of the 16 respondents used at least two thirds of the resource types (4 out of 6 resource types).

All but one of the respondents (15 out of 16) used dental images in their professional 
efforts, and the remaining respondent made use of other images.

Table 2: Resource Use by Respondent

\begin{tabular}{|c|c|c|c|c|c|c|c|}
\hline$\#$ & S. Engine & Univ. Resource & Non-Univ. Resource & Database & CD/DVD & Other & Total \\
\hline R01 & $x$ & $x$ & $x$ & $x$ & $x$ & $\mathbf{x}$ & 6 \\
\hline R02 & $x$ & $\mathbf{x}$ & $\mathbf{x}$ & $\mathbf{x}$ & $\mathbf{x}$ & $x$ & 6 \\
\hline R03 & & $x$ & & $x$ & & $x$ & 3 \\
\hline R04 & $\mathbf{x}$ & $x$ & $\mathbf{x}$ & $\mathbf{x}$ & $x$ & $x$ & 6 \\
\hline R05 & $\mathbf{x}$ & $x$ & $x$ & $\mathbf{x}$ & $x$ & & 5 \\
\hline R06 & $x$ & & $\mathbf{x}$ & $\mathbf{x}$ & $x$ & & 4 \\
\hline R07 & $x$ & $x$ & $x$ & $\mathbf{x}$ & $x$ & & 5 \\
\hline R08 & $x$ & $x$ & & $x$ & & $x$ & 4 \\
\hline R09 & $\mathbf{x}$ & $x$ & $x$ & $x$ & & & 3 \\
\hline R10 & $x$ & $x$ & & & $\mathbf{x}$ & & 3 \\
\hline R11 & $x$ & $x$ & & $\mathbf{x}$ & $x$ & & 4 \\
\hline R12 & $x$ & $x$ & & $\mathbf{x}$ & & & 3 \\
\hline R13 & $x$ & $x$ & & $\mathbf{x}$ & $x$ & & 4 \\
\hline R14 & $\mathbf{x}$ & & $\mathbf{x}$ & & $x$ & & 3 \\
\hline R15 & $x$ & $x$ & & $\mathbf{x}$ & $x$ & & 4 \\
\hline R16 & $x$ & & & & $\mathbf{x}$ & & 2 \\
\hline Total & 15 & 13 & 7 & 13 & 12 & 5 & \\
\hline
\end{tabular}

The semistructured components of the interviews yielded a large number of useful statements. The respondents made a total of 1,046 statements about the resource types in question, with a mean of 65.4 statements per respondent, a median of 61.5 statements per respondent, a low of 36 statements by respondent R10, and a high of 125 statements by respondent R02. Table 3 breaks the statements down by the resource referred to, and whether the statement was grouped under Prompt, Hindrance, or Description. For example (under Engine), respondents made 74 statements about Internet search engines that were grouped under Prompt, 54 statements about Internet search engines that were grouped under Hindrance, and 4 statements about Internet search engines that were grouped under Description.

Table 3: Frequency of Respondent Statements by Prompt, Hindrance, and Description 


\begin{tabular}{|l|c|c|c|c|c|c||c|c|c|}
\hline & Engine & UnivRes & $\sim$ UnivRes & Dbase & CD/DVD & Image & Diglmage & $\sim$ Diglmage & Other \\
\hline Prompt & 74 & 71 & 38 & 50 & 61 & 92 & 186 & 9 & 33 \\
\hline Hindrance & 54 & 31 & 17 & 32 & 46 & 31 & 83 & 10 & 15 \\
\hline Description & 4 & 12 & 7 & 0 & 4 & 35 & 39 & 2 & 10 \\
\hline Total & 132 & 114 & 62 & 82 & 111 & 158 & 308 & 21 & 58 \\
\hline
\end{tabular}

As mentioned earlier, this paper focused on the use of images, and the information on other online resources types will be used to establish context. For all resource types, statements grouped under Prompt constituted at least a plurality, except in the case of statements about non-digital images, where a plurality of statements was grouped under Hindrance. This constitutes a negative .9:1 ratio of statements grouped under Prompt to statements grouped under Hindrance. For digital images the ratio of statements grouped under Prompt to the statements grouped under Hindrance was strongly positive, 2.2:1. For general references to images in which the respondent did not indicate the format in which the image came, the ratio of statements grouped under Prompt to the statements grouped under Hindrance was a strongly positive 3:1. The respondents also made a number of statements grouped under Description, but by definition, those statements did not include any indication as to why an image was used or not used. Those statements will not be addressed in this paper.

Of the 16 respondents, 9 either stated a direct preference for digital images, or criticized physical slides used with a traditional projector. One respondent referred to using physical slides when no other form was available. No respondent indicated a preference for physical slides.

General image use tended to be prompted either by teaching or use for writing articles, with teaching predominating. The images used tended to depict diseased tissue, dental procedures such as filling cavities, or the use of dental materials. For example, respondent R02 made a comment that effectively summed up general image use among the respondents, "I use these images all the time in my courses that I teach."

Hindrances in general image use also fell into several clear groups. One set of reasons centered on poor image quality and difficulty in creating suitable images. A number of respondents also mentioned lack of information to go with the images. Respondent R01 indicated "You can find specific images of disease states or specific things, but in terms of a case study, those are more difficult to find." The same respondent, along with other respondents, also mentioned legal hurdles, saying, "If I have a full patient view, with the regulations, I'm not going to share those images any more." 
The use of digital images also tended to be prompted by teaching and the writing of articles, but a number of respondents also mentioned using digital images with patients. For example, respondent R07 mentioned showing images to "prospective patients or to show the patient that the pictures are taken of before and after [a dental procedure]." Respondents also spoke favorably of the ease-of-use and manipulability of digital images. Respondent R09 voiced a general preference for digital images over non-digital images that was similar to the preference stated by other respondents: "If I can get the images for a PowerPoint through digital imaging, and use it in my PowerPoint, I do it that way." Respondent R03 stated "The storage of them [digital images] is immensely more compact." Respondent R03 also referred specifically to manipulating images: "You can revise them... You can add text."

Hindrances in using digital images also fell into several clear groups. Intellectual property rights were mentioned as hindering the use of digital images. For example, respondent R16 referred to weighing the use of using his or her own images against using images created by someone else: "you use theirs or you're probably safer legally to make them yourself although it does take more time if you have time." Respondents also voiced concern over ease-of-use issues and image quality. Respondent R15 expressed concern over software use: "I'm not really well-versed in say, Adobe Photoshop, or things like that." Respondent R08 indicated that not all of the available digital images were of sufficient quality: "sometimes the pixel range is such that you can't enlarge them as you'd like."

It was only in statements describing non-digital image use that the respondents made fewer statements grouped under Prompt than under Hindrance (9 statements to 10). It is also worth noting that the use of non-digital images only prompted a total of 21 statements, as opposed to 158 statements for general image use and 308 statements for digital image use (see Table 3).

The use of non-digital images, when it occurred, tended to be prompted by issues of quality or lack of choice. Respondent R02 indicated that, "The physical slides, now those [digital images] getting better, those slides, but the physical is more live, you know, you see, especially if you take it directly from the patient." Respondent R01 referred to simple lack of other images: "It's only when I'm caught really short, or I need to something quickly, would I show the [physical] slides."

The hindrances in using non-digital images described by the respondents centered on simple lack of access to traditional slide projectors and lack of ability to manipulate non-digital images. Respondent R02 indicated that, "now it is inconvenient to use it [physical slides], because there is no more machines, but I have physical [slides]." 
Respondent R15 referred to that static nature of physical slides: "Once you have a slide you have a slide. You can't do anything about it."

One general pattern in the data from the interviews is worth mentioning at this point. An even half of the respondents (8 of 16) mentioned case studies, before and after series, or accompanying information such as annotations as being attributes that would serve as positive prompts to image use. For example, respondent R12 expressed a desire for images that included diagnoses, for example, "...if you do not have it in your own file, and if they have a better picture than you do have it, with the diagnosis written on there." Respondent R02 referred to a desire for images that showed a progression from healthy to diseased tissue. "This is to do with showing the etiology, the root cause of the problem." What is perhaps most interesting is that elicitation of these detailed statements was unplanned and emerged during the course of the interviews. We expected that respondents would mention organizational or descriptive aspects of image collections, and in keeping with the goals of the study we wanted to elicit such statements. But we did not expect the level of detail or variety mentioned by the respondents. This emerged early in the interviews, but in order not to bias the results, we did not alter the original interview instrument to elicit more of these detailed comments. Other respondents made less detailed comments that also referred to organizational aspects of digital image collections. For example, respondent R03 mentioned using a tooth atlas to show where particular tissue types were found. That suggests physical location in the body as one potentially useful metadata element.

\section{Survey of Academic Deans}

The academic deans from dental schools in the U.S. and Canada were invited via the ADEA listserv. Of the 56 Deans, 18 (32\%) responded. Table 4 shows the result by online resource addressed in the questionnaire.

Table 4: Frequency of Image Use and Sources for Images Based on Survey of ADEA Deans

\begin{tabular}{|l|c|c|c|c|c||c|c|c|}
\hline $\begin{array}{c}\text { Frequency/ } \\
\text { Resource }\end{array}$ & $\begin{array}{c}\text { Digital } \\
\text { Images }\end{array}$ & $\begin{array}{c}\text { Internet } \\
\text { (Free) }\end{array}$ & $\begin{array}{c}\text { Personal } \\
\text { Collection }\end{array}$ & $\begin{array}{c}\text { Subscription } \\
\text { Resources }\end{array}$ & $\begin{array}{c}\text { Digital } \\
\text { Textbooks }\end{array}$ & $\begin{array}{c}\text { Digital } \\
\text { Journal } \\
\text { Articles }\end{array}$ & $\begin{array}{c}\text { Commercial } \\
\text { CD/DVD }\end{array}$ & $\begin{array}{c}\text { Additional } \\
\text { Images }\end{array}$ \\
\hline $\begin{array}{l}\text { Frequency } \\
(\mathrm{n}=18)\end{array}$ & 18 & 12 & 17 & 0 & 8 & 6 & 13 & 18 \\
\hline
\end{tabular}

All of the respondents (18 of 18) indicated that faculty members at their school used digital images in teaching dentistry. When asked whether their faculty members would 
benefit from and use a source of digital images available over the Internet, all of the respondents (18 of 18 ) answered affirmatively.

The respondents also indicated that faculty members at their institutions used 5 of the 6 listed resources types in gathering digital images for their use. Substantial majorities indicated faculty members at their institutions use the Internet (12 of 18), personal collections (17 of 18), and CD-ROMs or DVDs (12 of 18). Every respondent indicated that faculty members at his or her institution gathered digital images from at least one of the sources.

\section{Conclusions}

\section{Interviews with Faculty members and Clinicians}

The use of online resources is deeply embedded in the working lives of both the faculty members and clinicians. A substantial number of the respondents indicated a preference for digitized images, and no respondent indicated an overall preference for physical slides. The use of physical slides was restricted to particular, often nonfavorable, situations such as an instance in which a digitized slide was simply unavailable. This suggests that an online repository of digitized dental images will constitute a good match with the way in which they gather information for use in teaching, research, and clinical practice. The use of such a broad range of online resources clearly helped establish the appropriateness of online delivery for faculty members and clinicians.

The use of dental images more specifically is also deeply embedded in the working lives of both the faculty members and clinicians. Virtually all of them made some use of dental images, and the data strongly suggest that they prefer to use images in digitized form rather than in the form of traditional projected slides. The respondents voiced relatively negative views of non-digital images, and the patterns in the statements made it clear that use of digital images predominates, and predominates by choice. While the respondents did not generally indicate a lack of images, they did express a desire for higher quality, manipulable images that would add depth and variety to existing collections. That is a need that can be directly addressed by the creation of an online repository of digitized dental images. In the context of the first goal of the study, online delivery of digitized dental images matches the needs of dental faculty members and clinicians.

The interviews also made it apparent that a metadata scheme for describing the digitized images would be helpful. Half of the respondents (8 of 16) made statements that referred to such features as accompanying case studies, annotations, or before and after 
sequences to illustrate treatments. Other respondents made less detailed statements that also indicated possible metadata elements such as the name of a disease or injury, and location in the maxillofacial region. All of those features could be delivered or described through a concept-based metadata scheme. Other respondents referred to color within the images, which could be made searchable through content-based image retrieval. These potential metadata elements were not formally ranked by frequency because the interview instrument was not designed to allow that. A follow-up study is in progress which will provide a more formal examination of possible specific elements for a metadata scheme. In the context of the second goal of the study, a metadata scheme for content-based image retrieval would match the needs of the faculty members and clinicians. Eventual development of content-based image retrieval might also usefully match the respondents' needs, but concept-based metadata for retrieval would seem to be a better match.

\section{Survey of Academic Deans}

The survey of academic deans at dental schools from across the U.S. and Canada provided validation and national context for the interviews with faculty members and clinicians at the University at Buffalo's School of Dental Medicine. All of the deans indicated that their faculty members use digital images, and all of the deans also indicated that a repository of additional images would be useful for their faculty members. This strongly suggests that the needs elicited during the individual interviews have importance that extends beyond the particular school from which the sample is drawn.

\section{General Conclusions}

At a minimum, the results suggested that further research toward a productive role for an online repository of digitized dental images will be worthwhile. Current collections of digitized dental images fill many, but not all, of the needs of dental faculty members and clinicians. A repository of such images, available for academic use, would help faculty members and clinicians by providing a practical source for a variety of high-quality, manipulable images that would further their research and clinical efforts. A concept-based metadata scheme would play a positive role in making the digitized dental images in the repository more useful.

\section{Limitations}


As with any study, there are several limitations to the needs assessment described in this paper.

The principal limitation lies in the size of the sample, which necessitates great care in qualifying the conclusions reached in this study. The purposive nature of the sample helped to reduce potential problems by including a variety of perspectives from faculty members and clinicians who work in different aspects of dentistry. Nonetheless, the conclusions in this study need to be viewed as preliminary conclusions subject to later confirmation. Given the broad range of specialties within dentistry covered by the sample, though, it is unlikely that the results of this study will be substantially overturned.

Similar potential problems stemmed from the fact that the sample for the interviews was drawn from a single dental school. The University's School of Dental Medicine, however, serves as an adequate representative of U.S. and Canadian dental schools more generally because it has a broad dental curriculum and utilizes resources similar to those utilized at other dental schools across the country.

Finally, the survey of academic deans at dental schools also has limitations. While the academic deans are in a position to know what resources, in general, their faculty members use, the evidence gathered from the survey is nonetheless at least partially indirect. This is partially compensated for by the fact that academic deans from dental schools across the U.S. and Canada were invited to participate. The results from the survey of academic deans thus serve to reinforce the preliminary value of the conclusions drawn from data gathered during the individual interviews.

\section{Acknowledgments}

This paper is based in part on a poster presented at the ASIS\&T 2005 Annual Meeting.

\section{References}

Armitage, L. \& Enser, P. G. B. (1997) Analysis of user need in image archives Journal of Information Science 23, 287-299

Attig, J., Copeland, A., \& Pelikan, M. (2004) Context and meaning: The challenges of metadata for a digital library within the university College and Research Libraries 65, 251-261

Carletta, J. (1996) Assessing agreement on classification tasks: The kappa 
Choi, Y. \& Rasmussen, E. (2003) Searching for images: The analysis of user's queries for image retrieval in American History Journal of the American Society for Information Science 54(6), 498-511

Chu, H. (2001) Research in image indexing and retrieval as reflected in the literature Journal of the American Society for Information Science 52(12), 1011-1018

Cohen, J. (1960) A coefficient of agreement for nominal scales Educational and Psychological Measurement 20(1), 37-46

Corridoni, J.M., Del Bimbo, A, \& Vicario, E. (1998) Image retrieval by color semantics with incomplete knowledge Journal of the American Society for Information Science 49(3), 267-282

Del Bimbo, A. (1999) Visual information retrieval San Francisco, CA: Academic Press Fidel, R. (1997) The image retrieval task: Implications for the design and evaluation of image databases The New Review Hypermedia and Multimedia 181-199

Fidel, R., Hahn, T.B., Rasmussen, E. M. \& Smith, P. J., eds. (1994) Challenges in Indexing Electronic Text and Images Medford, N. J.: Learned Information for the American Society for Information Science

Fiuza, M., de Padua F., Lopes, M.G. (1997) The digital image archive-its clinical applications and importance in cardiology consulting by telemedicine Revista Portuguesa de Cardiologia 16(2), 127-139

Fleiszer, D. M., Posel, N.H. \& Steacy, S. P. (2004) New directions in medical e-curricula and the use of digital repositories Academic Medicine 79(3), 229-235

Goldberg L. J., Ceusters W., Eisner J. \& Smith B. (2005) The significance of SNODENT In Engelbrecht R. et al. (Eds.) Medical Informatics Europe pp. 737-742. Amsterdam: IOS Press

Goodrum, A. (1997) Evaluation of text-based and image-based representations for moving image documents Ph.D. Dissertation, University of North Texas, Denton, TX

Goodrum, A. \& Spink, A. (2001) Image searching on the Excite web search engine Information Processing and Management 37(2), 295-312

Gudivada, V. \& Raghavan, V. (1995) Content-based image retrieval systems Computer 29, 9, 18-22

Hastings, S. K. (1994) An exploratory study of intellectual access to digitized art 
images Unpublished doctoral dissertation, Florida State University, Tallahassee

Hastings, S. K. (1995) Query categories in a study of intellectual access to digitized art images Proceedings of the 58th Annual Meeting of the American Society for Information Science 3-8

Jasco, P. (1998b, Nov./Dec.) Searching for images by similarity online Online 22, 99-102+

Jasco, P. (1998a, June) Searching for video on the World Wide Web Information Today 15, 40- 44

Jorgensen, C. (1996) Indexing images: Testing an image description template Proceedings of the 59th Annual Meeting of the American Society for Information Science 209-213

Jorgensen, C. (1998) Attributes of images in describing tasks Information Processing and Management 34(2/3), 161-174

Jorgensen, C. Jaimes, A., Benitez, A.B. \& Chang, S. (2001) A conceptual framework and empirical research for classifying visual descriptors Journal of the American Society for Information Science 52(11), 938-947

Jorgensen, C. \& Jorgensen, P. (2003) Image querying by image

professionals Proceedings of the 66th Annual Meeting of the American Society for Information Science 349-356

Keister, L.A. (1994) User types and queries: Impact on image access systems Challenges in Indexing Electronic Text and Images Medford, N. J.: Learned Information for the American Society for Information Science, pp. 7-22

Lawrence, J. C., \& Levy, L. S. (2004) Comparing the self-described searching knowledge of first-year medical and dental students before and after a MEDLINE class Medical Reference Services Quarterly 23(1), 73-81

Li, S., Lee, M. \& Adjeroh, D. (2005) Effective invariant features for shape-based image retrieval Journal of the American Society for Information Science 56(7): 729-740

Narasimhalu, A. (1995) Special section on content-based retrieval Multimedia Systems 3, 1, 1-12

O'Connor, B. C. (1996) Explorations in indexing and abstracting: Pointing, virtue and power Englewood, CO: Libraries Unlimited

O'Connor, B., O'Connor, M., \& Abbas, J. (1999) User reactions as access mechanism: An 
exploration based on captions for images Journal of the American Society for Information Science 50(8), 681-697

Ornager, S. (1997) Image retrieval: Theoretical analysis and empirical user studies on accessing information in images. Digital collections: Implication for users, funders, developers, and maintainers Proceedings of the 60th Annual Meeting of the American Society for Information Science 202-211

Paling, S., and Miszkiewicz, M. (2005, October) Digital resources for dentistry database: A needs assessment Poster presented at the ASIS\&T annual meeting, Charlotte, NC

Paling, S., Miszkiewicz, M., Abbas, J., and Zambon, J. (In progress) Digital Image Use in Dental Education: Establishing Current Context and Future Needs

Pu. H.T. (2003) An analysis of web image queries for search Proceedings of the 66th Annual Meeting of the American Society for Information Science 340-348

Rasmussen, E. (1997) Indexing images Annual Review of Information Science and Technology 32, 169-196

Rowe, N. (1994) Inferring depictions in natural-language captions for efficient access to picture data Information Processing and Management 30(3), 379-388

Schleyer, T.K., Spallek H., Bartling, W.C., \& Corby, P. (2003, January) The technologically well-equipped dental office Journal of the American Dental Association 134(1): 30-41

Smeulders, A.W.M., Worring, M., Santini, S., Grupta, A., \& Jain, R. (2000) Content-based image retrieval at the end of the early years IEEE Transactions on Pattern Analysis and Machine Intelligence 22(12): 1349-1380

Smith, J.R. (2001) Quantitative assessment of image retrieval effectiveness Journal of the American Society for Information Science 52(10), 969-979

Tsafrir, J., Ohry, A. (2001) Medical illustration: from caves to cyberspace Health Information \& Libraries Journal 18(2), 99-109

Turner, J. (1997) Comparing user-assigned terms with indexer-assigned terms for storage and retrieval of moving images: Preliminary results Proceedings of the 59th Annual Meeting of the American Society for Information Science pp. 214-217

University of Texas Health Science Center at San Antonio, Office of Educational Research and Development. (2003) IREC phase one report Retrieved February 12, 2006, from http://www.uthscsa.edu/ais/erd/documents/irecphaseonereport.pdf

Zachary, J. \& lyengar, S.S. (2001) Information theoretic similarity measures for content 
based image retrieval Journal of the American Society for Information Science 52(10), 856-857 